\title{
Heat shock protein 27 downstream of P38-PI3K/Akt signaling antagonizes melatonin-induced apoptosis of SGC-7901 gastric cancer cells
}

Wenjie Deng ${ }^{1,2}$, Yujie Zhang ${ }^{1}$, Luo Gu ${ }^{1,2,3}$, Jie Cui ${ }^{3}$, Biao Duan², Yueyuan Wang ${ }^{2}$ and Jun Du 1,2*

\begin{abstract}
Background: Despite the fact that melatonin treatment shows some promise in gastric cancer, the molecular mechanisms of gastric cancer cells in response to melatonin remains to be determined.

Methods: The SGC-7901 gastric cancer cells were treated with different concentrations of melatonin for 24 and $48 \mathrm{~h}$. Cell viability was determined by MTT assay, Hoechst 33258 staining and FACS analysis were used to detect apoptotic cells. The contents and activation of apoptosis-related proteins HSP27, Akt and P38 were evaluated by immunoblotting analysis. Then we treated SGC-7901 cells with HSP27-specific siRNA, PI3K inhibitor LY294002 or P38 inhibitor SB203580 to investigate the role of HSP27, Akt and P38 in the anti-apoptotic response of SGC-7901 cells to melatonin.

Results: Melatonin suppressed cell viability and stimulated apoptosis of gastric cancer SGC-7901 cells dosedependently. Mechanistically, the observed apoptosis was accompanied by the melatonin-induced phosphorylation of HSP27. HSP27-specific siRNA transfection effectively reduced HSP27 phosphorylation and augmented melatonininduced apoptosis, indicating that HSP27 is resistant to melatonin-induced apoptosis. Moreover, melatonin increased PI3K/Akt activation, LY294002 abrogated HSP27 activation and promoted cell apoptosis induced by melatonin. Furthermore, melatonin increased P38 activity, and P38 inhibitor SB203580 inhibited melatonin-induced PI3K/Akt, HSP27 activation and accelerated cell apoptosis.
\end{abstract}

Conclusion: In contrast to the well-established anti-cancer properties of melatonin, our study revealed clearly a distinguishable anti-apoptotic pathway induced by melatonin, that is, HSP27 plays a crucial role in apoptotic resistance in melatonin-treated gastric cancer cells, and its activation is most likely via the activation of P38/PI3K/Akt signaling.

Keywords: Melatonin, P38, PI3K/Akt, HSP27, Gastric cancer

\section{Background}

Gastric cancer has become the fourth most common malignancy and the second in mortality of total cancer worldwide [1]. It is estimated that in 2012 there were 951,600 new cases and 723,100 deaths from gastric cancer in the world [2]. Despite the development of therapeutic intervention for gastric cancer in clinical

\footnotetext{
*Correspondence: dujun@njmu.edu.cn

2 Department of Physiology, Nanjing Medical University, 140 Hanzhong

Road, Nanjing, Jiangsu 210029, China

Full list of author information is available at the end of the article
}

trials, drug resistance is still the major reason why failure occurs in gastric cancer treatment. As reviewed recently, melatonin, an indolamine derived from the L-tryptophan, has been shown to exert important protective effects in the gastrointestinal tract [3]. Although further study in additional settings and populations is required, melatonin is of great interest among the compounds tested as a potential therapy in gastric cancer.

Melatonin is an endogenous hormone that is required for the daily onset of darkness. Besides its use in the treatment of some sleep disorders, melatonin has been characterized as possessing a pro-apoptotic role in the 
treatment of cancer, and there seems to be some promise in several types of cancers such as breast, prostate as well as colorectal cancer [4-6]. There is at least 400 times more melatonin produced in the gastrointestinal tract than in the pineal gland [7], and melatonin has been demonstrated to suppress gastrointestinal carcinogenesis under some circumstances such as chronic inflammation $[8,9]$. The safety of melatonin heralds it as a great prospect for gastrointestinal cancer therapy [3], however, the molecular mechanism related to its effect on gastric cancer remains unelucidated.

HSP27, a member of the small heat shock protein (HSP) family, is believed to have tumorigenic and prometastatic functions, characterized by its dynamic phosphorylation leading to heterogeneous oligomerization under different conditions such as oxidative stress, heat shock as well as chemical stress [10]. In fact, epidemiologic surveys have demonstrated that HSP27 is upregulated in many cancers including ovarian cancer, colorectal cancer and gastric cancer, where it has been identified as a tumor prognostic marker [11-13]. HSP27 is also closely correlated with increased resistance to apoptosis in stressed cancer cells. For example, HSP27 accumulation reduces the chemosensitivity induced by vincristine and adriamycin agents in gastric cancer cells [14]. In contrast, a combination of traditional chemotherapeutic agents (cisplatin, gemcitabine) and HSP27 inhibitor (quecertin) exerts a surprising greater chemotherapeutic effect in lung stem like cells [15]. Thus, it is worthwhile to explore whether HSP27 is involved in melatonin-induced apoptosis of gastric cancer cells.

Recent studies from our laboratory showed that HSP27 phosphorylation could be induced by P38 activation and phospho HSP27 acts as a regulator of cell cytoskeleton reorgnization and cell adhesive ability [16]. In addition, HSP27 has been found to direct chaperoning interaction with Akt, and protest adenocarcinoma cells from UV-induced apoptosis [17]. Based on above information, we focused this study on HSP27 regulation of apoptosis induced by melatonin, and in an attempt to gain further mechanistic insights into the molecular pathways leading to melatonin-induced alteration of HSP27 phosphorylation in SGC-7901 gastric cancer cells.

\section{Methods}

\section{Cell culture}

Human gastric cancer cell line SGC-7901 was obtained from the Cell Biology Institute of Chinese Academy of Sciences (Shanghai, China). SGC-7901 cells were cultured in Dulbecco's modified Eagle's medium (DMEM, high glucose) (Hyclone, Thermo Scientific, Waltham, MA, USA) supplemented with $10 \%(\mathrm{v} / \mathrm{v})$ fetal bovine serum (FBS) (Hyclone) and antibiotics $(100 \mathrm{U} / \mathrm{mL}$ streptomycin and $100 \mu \mathrm{g} / \mathrm{mL}$ penicillin) (Invitrogen, USA) in a humidified incubator at $37^{\circ} \mathrm{C}$ with $5 \% \mathrm{CO}_{2}$. Cells were grown on coverslips for fluorescence staining and on plastic dishes for protein extraction.

\section{Treatment and transfection}

Melatonin (Sigma, St Louis, MO, USA) was dissolved in ethanol and cells were treated with melatonin for the indicated times and doses. In experiments to determine the effects of inhibitors, LY294002 (Sigma) and SB203580 (Beyotime, Nantong, China) on cell growth inhibition and apoptosis, cells were treated with these kinase inhibitors for $30 \mathrm{~min}$ prior to melatonin treatment.

The sequences of small interfering RNA (siRNA) for HSP27 was 5'-UGAGAGACUGCCGCCAAGUAA-3', the sequence of control siRNA was $5^{\prime}$-UUCUCCGA ACGUGUCACGUTT-3' (GenePharma Co., Shanghai, China). Cells were transfected with control siRNA or HSP27 siRNA with Lipofectamine 2000, according to the manufacturer's instruction.

\section{Immunoblotting analysis}

Subconfluent cells were washed with PBS, and lysed with RIPA lysis buffer $(150 \mathrm{mmol} / \mathrm{L} \mathrm{NaCl}, 50 \mathrm{mmol} / \mathrm{L}$ Tris$\mathrm{HCl}(\mathrm{pH} 7.4), 1$ \% Triton X-100, 1 \% sodium deoxycholate, $0.1 \%$ SDS) with $1 \mathrm{mM}$ sodium orthovanadate, $1 \mathrm{mM}$ PMSF, and $1 \%$ cocktail of protease inhibitors (Sigma). The lysates were clarified by centrifugation at $12,000 \mathrm{~g}$ for $20 \mathrm{~min}$ at $4{ }^{\circ} \mathrm{C}$ and separated by SDS-PAGE followed by transfer onto nitrocellulose membranes. The following antibodies were used: rabbit anti-P38 antibody, rabbit anti-P-P38 antibody, rabbit anti-P-Akt antibody, mouse anti-HSP27 antibody and rabbit anti-P-HSP27 antibody (Cell Signaling, Danvers, MA, USA), rabbit anti-Akt antibody (Bioworld, Louis Park, USA), rabbit anti-GAPDH antibody (Santa Cruz, CA, USA). Protein bands were detected by incubating with horseradish peroxidase-conjugated secondary antibodies (Santa Cruz) and visualized with ECL reagent (Millipore, Billerica, MA, USA). Digital images of immunoblots were obtained with a Chemidoc XRS and analyzed using the image analysis program Quantity One (Bio-Rad, Hercules, CA, USA).

\section{Hoechst staining}

Hoechst 33258 dyes (Beyotime) are cell permeable nucleic acid stains, which are useful for the recognition of DNA damage and cell apoptosis by monitoring the emission spectral shifts of the dyes. Cells were stained with Hoechst $33258(5 \mu \mathrm{g} / \mathrm{mL})$ in PBS for $30 \mathrm{~min}$ at room temperature, and then washed to remove unbound dye. Observation and photography was performed in a fluorescence microscope (Olympus BX 51, Tokyo, Japan). 


\section{Flow cytometry analysis}

Cells were trypsinized and resuspended in $1 \times$ binding buffer, double-stained with Annexin V-FITC and propidium iodide (Beyotime) at room temperature for $15 \mathrm{~min}$ in darkness. Subsequently, the stained cells were analyzed by flow cytometry for apoptotic analysis according to the manufacturer's protocol.

\section{Cell viability assay}

Cell viability was determined by 3-(4,5-dimethylthiazol2-yl)-2,5-diphenyltetrazolium bromide (MTT) assay as described previously [18]. In brief, SGC-7901 cells were seeded at a density of $5 \times 10^{3}$ cells per well into 96 -well plate and treated with melatonin for the indicated times and doses. After culture, cells were washed, MTT was added and the plate was incubated in the dark for $4 \mathrm{~h}$, followed by measurement at $490 \mathrm{~nm}$ using a microplate absorbance reader (Bio-Tek, Elx800, USA). The percent cell viability was calculated as the absorbance of melatonin treated sample/control sample absorbance $\times 100 \%$.

\section{Statistical analysis}

Data were analyzed by Image J and statistical analyses were carried out using the SPSS software version 15.0 (SPSS Inc., Chicago, IL, USA). Student's $t$ test was used to analyze differences between two groups. Statistical significance was considered when $P<0.05$.

\section{Results}

Melatonin promotes gastric cancer cell apoptosis in vitro

To assess the apoptosis effects of melatonin on gastric cancer cells, a widely used human gastric cancer cell line, SGC-7901, was employed. Clear evidence for apoptosis was obtained by monitored by fluorescence microscopy after staining with Hoechst 33258. As shown in Fig. 1a, b, in the control group, SGC-7901 cells exhibited regular shaped nuclei. In comparison, numbers of shrunken cells with condensed or fragmented nuclei, characteristic of apoptotic cells, were significantly increased in $1 \mathrm{mM}$ melatonin treated cultures. We also treated cells with different doses of melatonin and cell viability was measured by MTT assay. As shown in Fig. 1c, the cell viability decreased gradually in a dose-dependent manner. We also assessed cell apoptosis in response to $1 \mathrm{mM}$ melatonin treatment at 24 and $48 \mathrm{~h}$, and SGC-7901 showed enhanced cell apoptosis in a time-dependent manner (Fig. 1d). These results showed that melatonin suppressed cell viability of SGC-7901 in vitro. The marked decrease of cell viability by $1 \mathrm{mM}$ melatonin treatment most likely reflected the induction of cell apoptosis by melatonin. Thus, $1 \mathrm{mM}$ melatonin was used for further apoptosis studies.

\section{Knockdown of HSP27 aggravates melatonin-induced cell apoptosis}

To explore the mechanism whereby melatonin stimulates gastric cancer cell apoptosis, we first examined endogenous HSP27 activation after melatonin treatment. Melatonin treatment resulted in a time-dependent increase in HSP27 activity, as determined by immunoblotting analysis with an antibody against the phosphorylated form of HSP27. In contrast, the levels of total HSP27 were constant at all these time points (Fig. 2a). We also treated cells with different doses of melatonin $(0.01-1 \mathrm{mM})$ for $24 \mathrm{~h}$, and found that melatonin dose-dependently activated HSP27 in SGC-7901 cells (Fig. 2b). To determine the involvement of HSP27 in melatonin stimulated apoptosis of gastric cancer cells, specific siRNA for HSP27 were applied to SGC-7901 cells. As shown in Fig. 2c, HSP27 siRNA greatly knocked down total and phosphorylated HSP27 expression. Treatment of cells with $1 \mathrm{mM}$ melatonin increased the apoptosis of SGC-7901 cells when compared with control cells. As expected, HSP27 knockdown not only resulted in a significant increase of melatonin-stimulated cell apoptosis, but also induced cell apoptosis in the absence of melatonin treatment in SGC-7901 cells (Fig. 2d, e). These results indicate that the knockdown of HSP27 may aggravate both basal and melatonin-stimulated gastric cancer cell apoptosis.

\section{PI3K/Akt mediates HSP27-induced resistance to apoptosis induced by melatonin}

To determine whether PI3K/Akt is the upstream mediator of HSP27 activation by melatonin in our system, immunoblotting analysis of P-Akt (Ser473), a well accepted downstream target of PI3K, was used to determine the PI3K activity $[19,20]$. The results revealed a dose-dependent increase in PI3K activity following melatonin treatment. Immunoblotting analysis showed that the amount of phosphorylated Akt was increased significantly after melatonin stimulation with maximal activation at $1 \mathrm{mM}$ (Fig. 3a). To determine whether melatonin-stimulated HSP27 activity is PI3K/Aktdependent, we blocked PI3K/Akt activity by treating the cells with LY294002, a PI3K inhibitor (Fig. 3b), and examined HSP27 activity after stimulation with melatonin. The results showed that pretreatment with $10 \mu \mathrm{M}$ LY294002 largely inhibited melatonin-induced Akt and HSP27 phosphorylation in comparison with control cells (Fig. 3c). The effect of PI3K inhibitor on cell apoptosis was also investigated using Hoechst 33258 assay. Pretreatment with $10 \mu \mathrm{M}$ LY294002 resulted in a remarkable enhancer of both basal and melatonin-promoted cell apoptosis (Fig. 3d). These results suggest that PI3K/Akt acts upstream of HSP27 in resisting melatonin-stimulated gastric cancer cell apoptosis. 


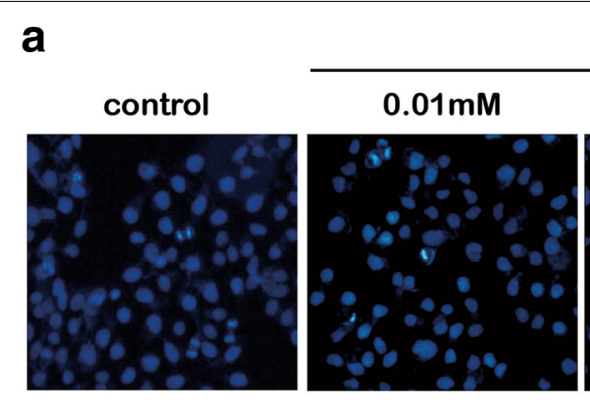

melatonin
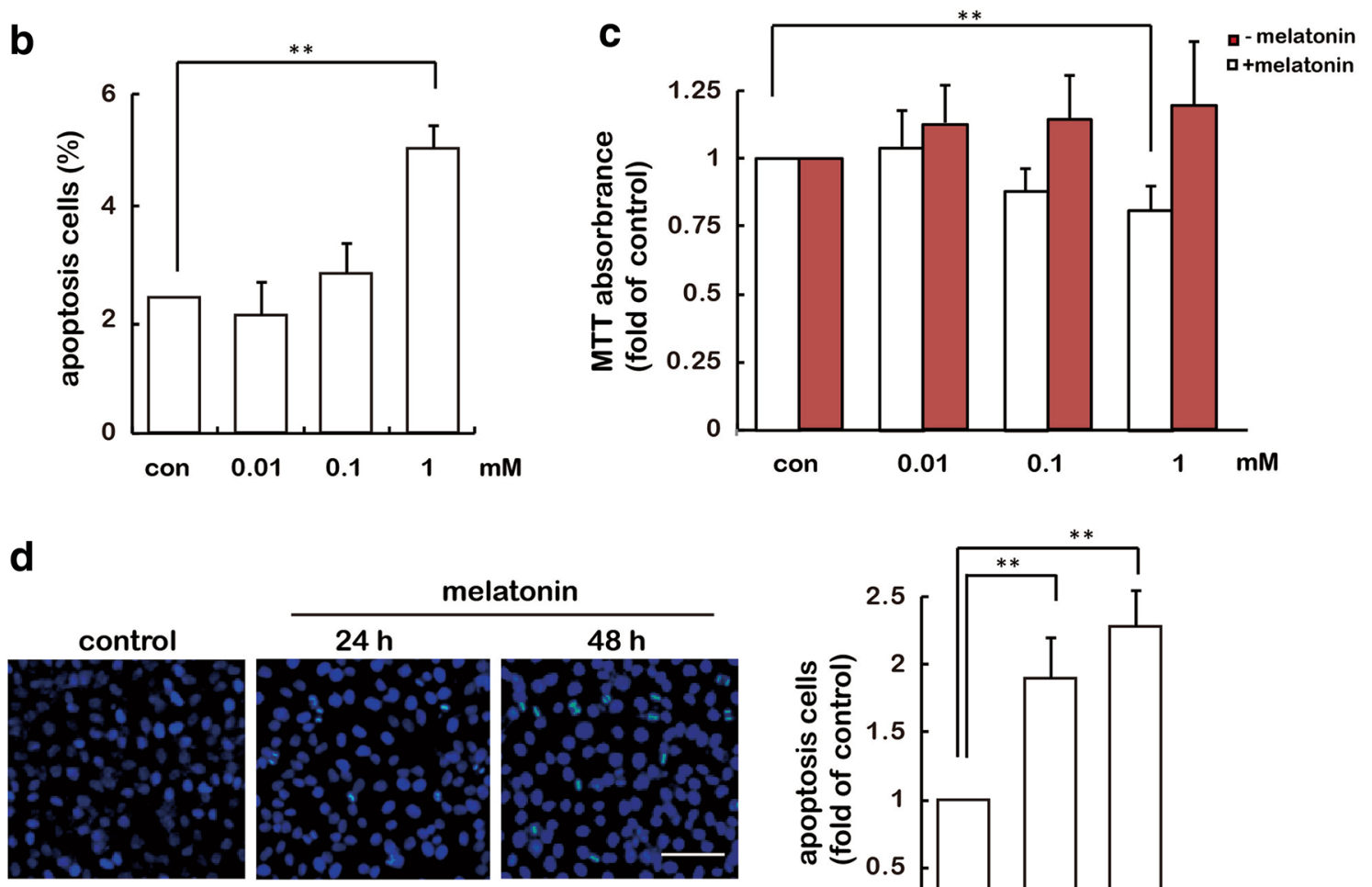

melatonin
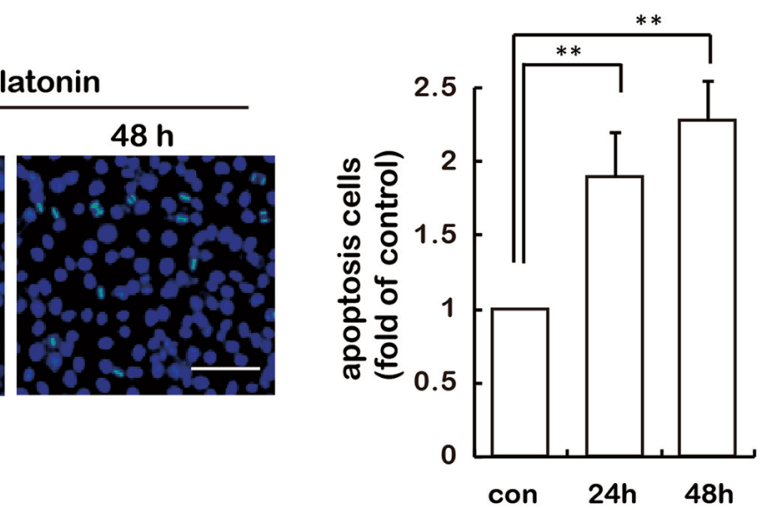

Fig. 1 Melatonin promotes apoptosis of SGC-7901 gastric cancer cells. SGC-7901 cells were incubated with melatonin for the indicated doses and periods. a The morphological figures of apoptosis were monitored by fluorescence microscopy after staining with Hoechst 33258 . Images are representative of at least three independent determinations. Scale bar 50 mm. b Melatonin-induced apoptosis of SGC-7901 gastric cancer cells. Values were presented as mean \pm SD of three independent experiments. c Cell proliferation was measured by MTT assay. $\mathbf{d}$ Apoptosis was evaluated for up to $48 \mathrm{~h}$ by Hoechst 33258 staining. Images are representative of at least three independent determinations. Scale bar 50 um. ${ }^{* * P}<0.01$

P38 activation is required for melatonin-induced PI3K/Akt/ HSP27 activation

We also examined the effect of melatonin on P38 activation in cultured gastric cancer cells. Melatonin treatment resulted in a dose-dependent increase in P38 activity, as determined by immunoblotting with an antibody against the phosphorylated form of P38 (Fig. 4a). To investigate whether P38 activation leads to melatonin-induced activation of the PI3K/Akt/HSP27 signaling pathway and cell apoptosis in cultured gastric cancer cells, SB203580, a known inhibitor of P38, was used and its effect on melatonin-induced PI3K/Akt/HSP27 activation was examined. Pretreatment with $20 \mu \mathrm{M}$ SB203580 remarkably abolished melatonin-induced PI3K/Akt (Fig. 4b) and HSP27 activation (Fig. 4c). These results indicate that melatonin can stimulate P38 activation in gastric cancer cells, and which may be an important mechanism for the stimulation of PI3K/Akt/HSP27 signaling pathway by melatonin.

Furthermore, by FACS analysis, we noticed that melatonin stimulated cell apoptosis was upregulated 

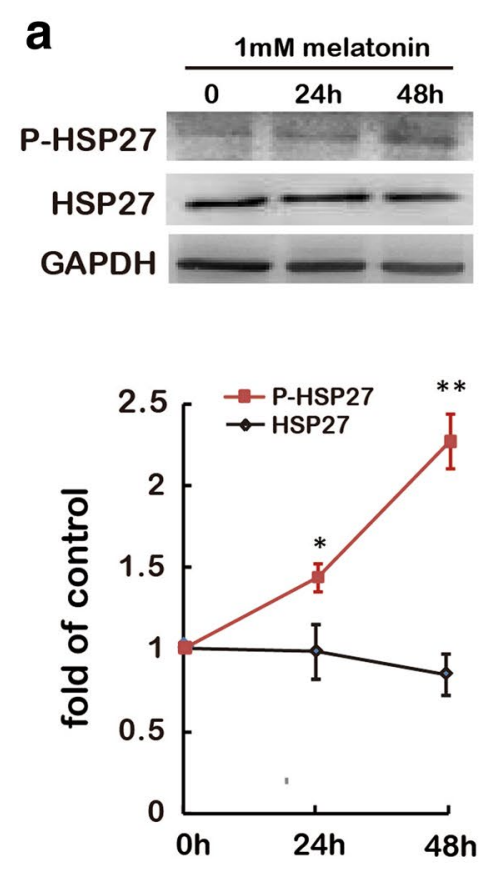

C

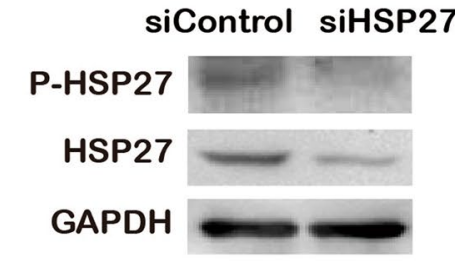

e

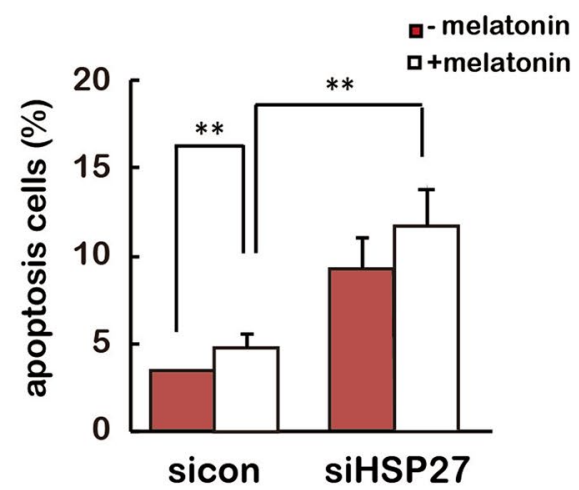

b
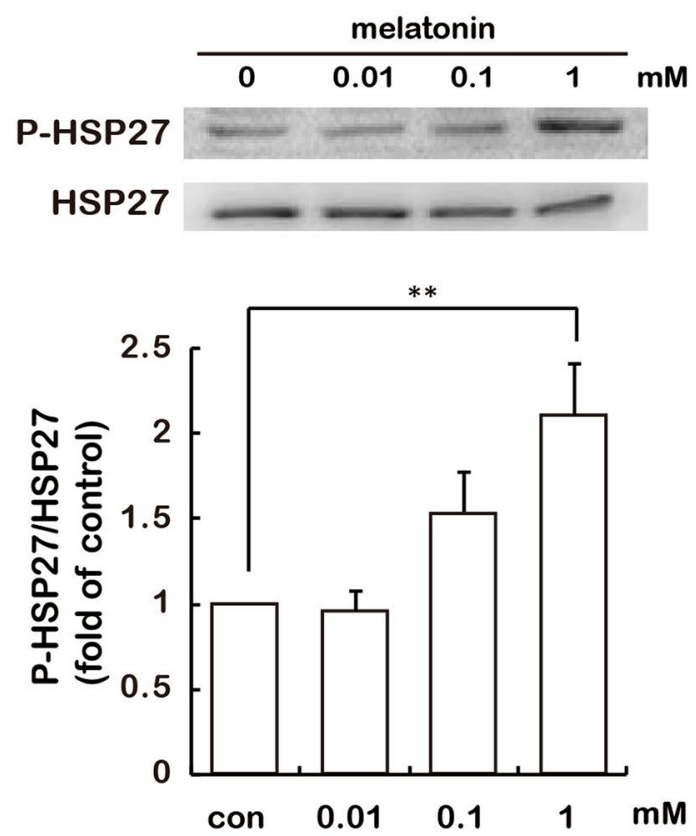

d

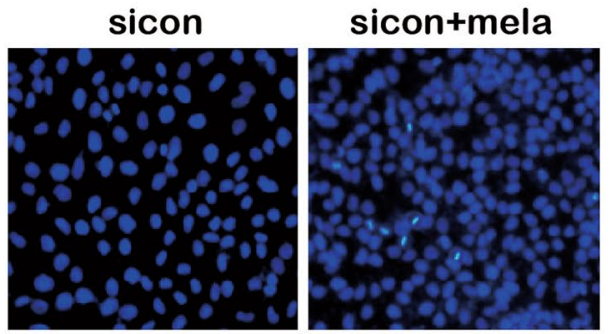

siHSP27

siHSP27+melatonin
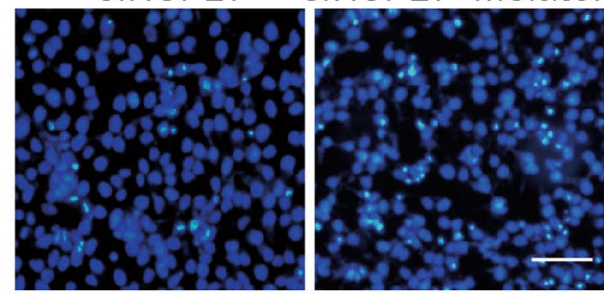

Fig. 2 HSP27 phosphorylation is required for resistance of apoptosis by melatonin. a, b SGC-7901 cells were treated with melatonin for the indicated periods (a) and doses (b), and HSP27 phosphorylation was determined by immunoblotting assay. GAPDH was used as a loading control. ${ }^{*} \mathrm{P}<0.05$, ${ }^{* *} \mathrm{P}<0.01$, referring to the difference between cells treated with and those without melatonin. c The expression of total and phosphorylation level of HSP27 after treatment with HSP27 siRNA. Total protein extracts from SGC-7901 cells transfected with HSP27 siRNA or control siRNA were analyzed by immunoblotting for P-HSP27 and HSP27. GAPDH was used as a loading control. d, e Cell apoptosis was determined by Hoechst 33258 staining in the SGC-7901 cells transfected with control siRNA or HSP27 siRNA. d Images are representative of at least three independent determinations. Scale bar $50 \mu \mathrm{m}$. e HSP27 siRNA treatment enhanced melatonin-induced apoptosis of SGC-7901 gastric cancer cells. Values were presented as mean \pm SD of three independent experiments. ${ }^{* *} \mathrm{P}<0.01$ 
a

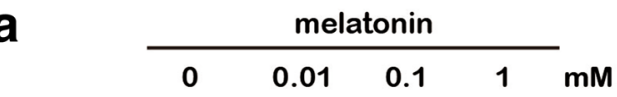

P-Akt

Akt

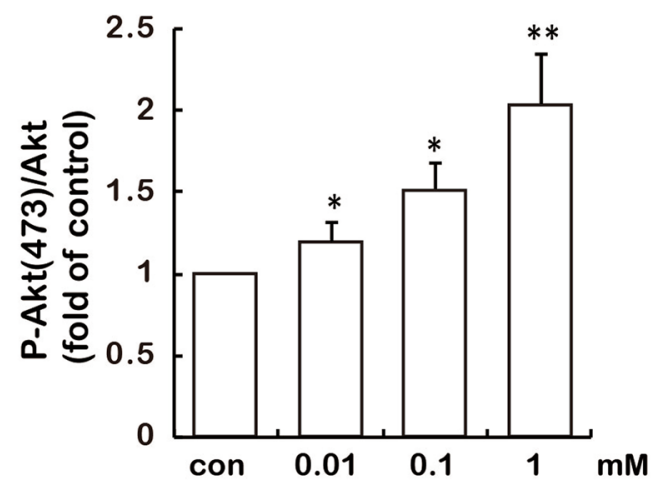

C
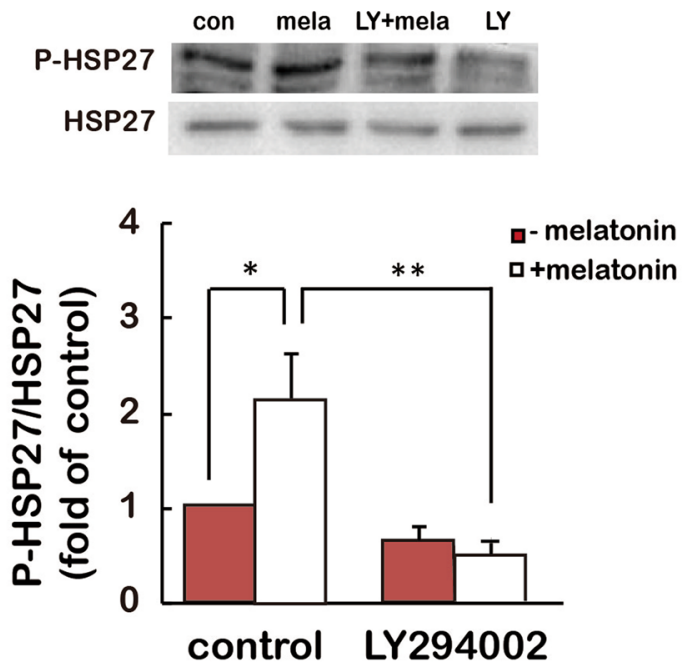

b
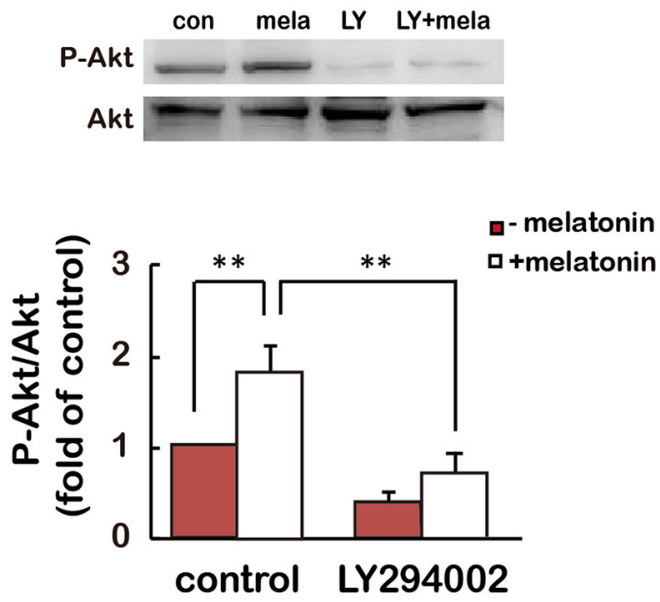

d ם-melatonin $\square+$ melatonin

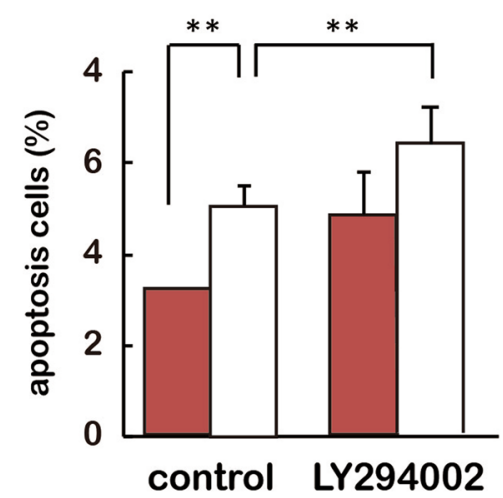

Fig. 3 HSP27 enhanced cell resistance to melatonin-induced cell apoptosis is mediated through PI3K/Akt. a, b Effect of melatonin stimulation on phosphorylation of Akt. SGC-7901 cells were treated with melatonin under different concentrations of melatonin for $24 \mathrm{~h}$. Melatonin-stimulated phosphorylation of Akt at Ser473 was determined by immunoblotting assay. GAPDH was used as a loading control. ${ }^{*} \mathrm{P}<0.05,{ }^{* *} \mathrm{P}<0.01$, referring to the difference between cells treated with and those without melatonin. b, c After treatment with $10 \mu \mathrm{M}$ PI3K inhibitor LY294002 for 30 min, SGC7901 cells were stimulated with $1 \mathrm{mM}$ melatonin for $24 \mathrm{~h}$ and then cells were analyzed by P-Akt (b) and P-HSP27 (c) expression, and $\mathbf{d}$ the effect of LY294002 on the melatonin-stimulated apoptosis in SGC-7901 cells was determined. *P $<0.05$, ${ }^{* *} P<0.01$

significantly by the pretreatment with LY294002 and SB203580, HSP27 knockdown also resulted in a significant increase of melatonin-stimulated cell apoptosis (Fig. 5a). In the meantime, we noted that HSP27 silencing not only has no effect on melatonin stimulated P-Akt (Fig. 5c) and P-P38 (Fig. 5d) level, but also induced P-P38 and P-Akt expression in the absence of melatonin treatment. These results indicate that the activation of HSP27 is not essential for both basal and melatoninstimulated gastric cancer cell P38 and PI3K/Akt activation. Together, all the data above suggest a model that melatonin induces P38 activation, which stimulated activation of PI3K/Akt/HSP27 signaling for anti-apoptosis initiation (Fig. 5e). 


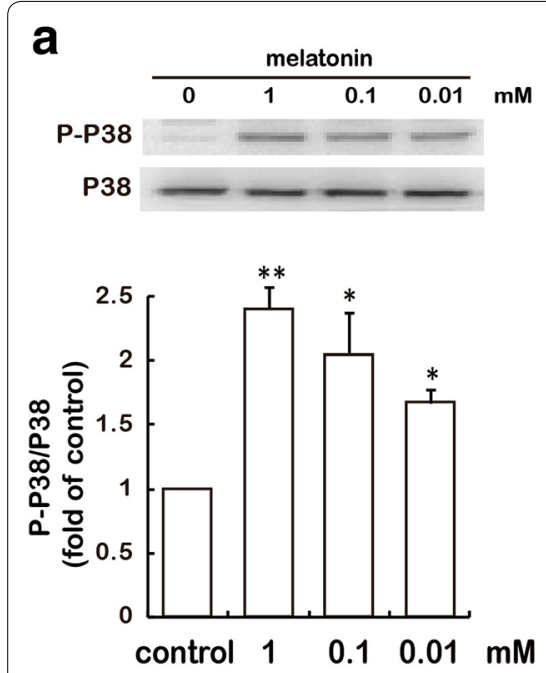

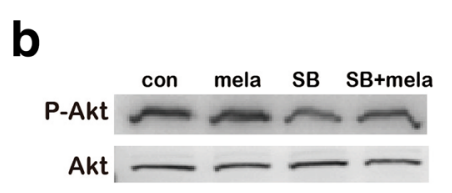
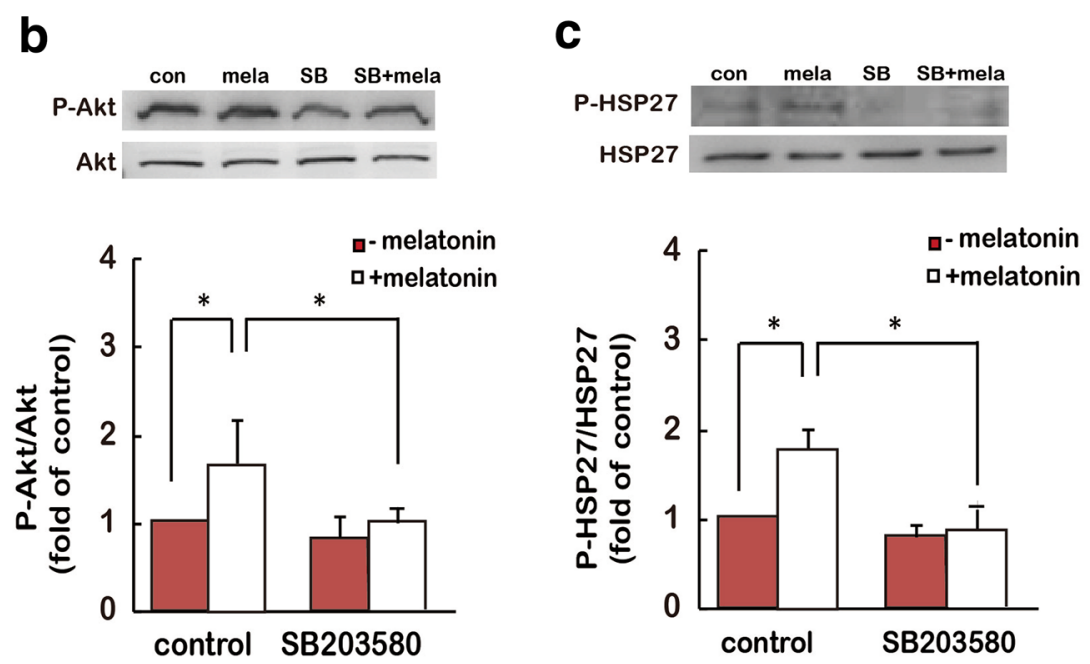

Fig. 4 P38 is required for the activation of PI3K/Akt and HSP27 by melatonin. a SGC-7901 cells were treated with melatonin for the indicated doses for $24 \mathrm{~h}$, and HSP27 phosphorylation was determined by immunoblotting assay. ${ }^{*} \mathrm{P}<0.05,{ }^{*} \mathrm{P}<0.01$, referring to the difference between cells treated with and those without melatonin. b, c After treatment with $20 \mu \mathrm{M}$ P38 inhibitor SB203580 for 30 min, SGC-7901 cells were stimulated with $1 \mathrm{mM}$ melatonin for $24 \mathrm{~h}$, then the cells were analyzed by P-Akt (b) and P-HSP27 (c) expression. ${ }^{*} \mathrm{P}<0.05$

\section{Discussion}

Besides its role in preventing angiogenic tumor cells dormancy [19], HSP27 is also essential in maintaining normal homeostasis and morphology of malignant cells in response to stress conditions. Previous studies have shown that the susceptibility of gastric ulceration induced by NSAID was reduced in the transgenic mice which were overexpressed HSP27 [20]. Although HSP27 phosphorylation contributes to arachidonic acid-induced apoptosis in brain endothelial cells [21], here, we reported that, in SGC-7901 gastric cancer cells, melatonin stimulated HSP27 phosphorylation dose-dependently. Importantly, depletion of HSP27 resulted in an increase in the number of melatonin-induced apoptosis cells, indicating that HSP27 is actively involved in maintaining homeostasis of SGC-7901 cells by inhibiting cell apoptosis. This is consistent with the observation that melatonin stimulates accumulation of nuclear phosphoHSP27 in human pancreatic carcinoma cells [22]. The reasons for these divergent cellular outcomes are presently unclear, but HSP27 interaction with distinct substrates, or binding partners after different stimulator treatment, might be able to explain the opposing responses on cell apoptosis. Actually, HSP27 could regulate apoptosis through an ability to interact with multiple points of the apoptotic signaling pathway. For example, the phosphoHSP27 directly interacts with Daxx, preventing the interaction of Daxx with both Ask1 and Fas that mediates cell apoptosis [23]. Collectively, these findings attest to phosphoHSP27 being a potentially resistant molecule in apoptosis induced by melatonin.
We next examined the potential activators for HSP27 in our system. Akt is a Ser/Thr kinase and downstream mediator of the PI3K pathway regulating cell survival, differentiation, and growth factor responsiveness. The frequent aberrant activation of the PI3K/Akt pathway in human cancer has made it an attractive therapeutic target [24]. In granular keratinocytes, Akt activation not only induced HSP27 phosphorylation, but also changes the equilibrium between cytoplasmic and nuclear phosphorylated HSP27, leading to its greater concentration in the cytoplasm [25]. In fact, it is reported that activation of Akt induced by phosphorylated HSP27 confers the apoptosis-resistance in t-AUCB-treated glioblastoma cells in vitro [26]. Our results show that melatonin induces a dose-dependent increase in PI3K/Akt activity. Blocking PI3K activity by LY294002 significantly prevents melatonin-induced HSP27 activation and aggravates cell apoptosis. Therefore, it may be reasonable to think that activation of PI3K/Akt signaling upon treatment with melatonin increased HSP27 phosphorylation, which was resistant to melatonin chemotherapy.

Several reports have demonstrated that HSP27 can form multicomponent complexes with Akt, and P38, and this complex has been shown to be involved in controlling HSP27 phosphorylation and stress-induced apoptosis [17, 27, 28]. As reported previously, activation of P38 was accompanied by Akt suppression in the apoptotic process in some types of cancer cells [29, 30]. Conversely, there also has a good evidence suggesting a link from MEKK4 and P38, via HSP27, to Akt activation, which is essential for the regulation of cell fate in 
a
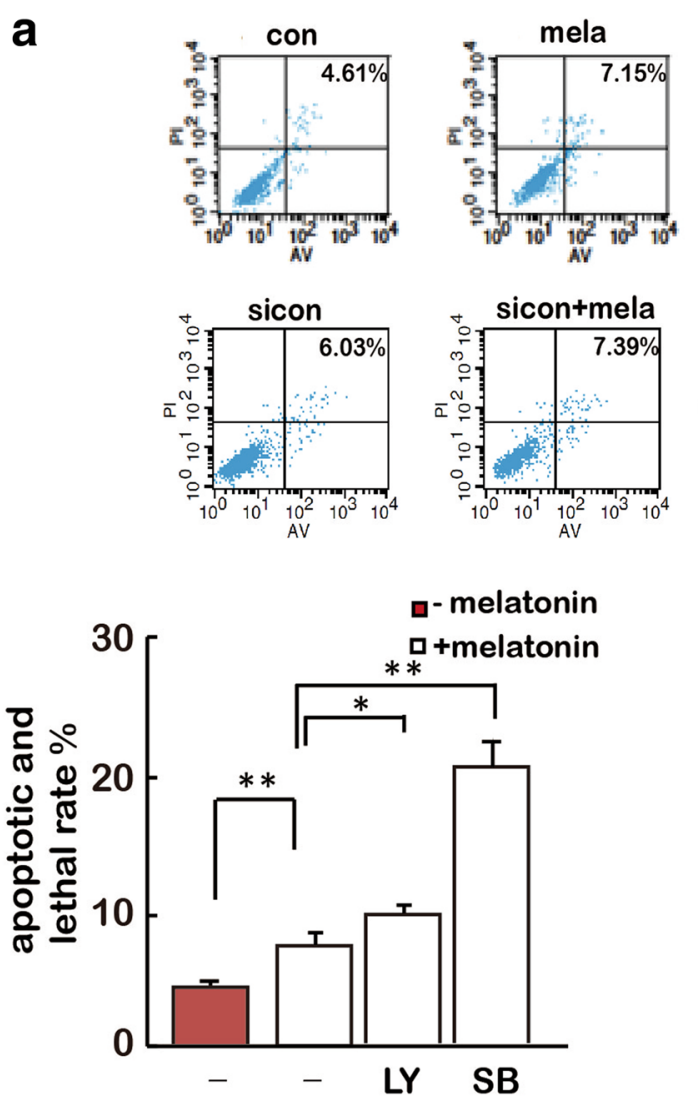

b
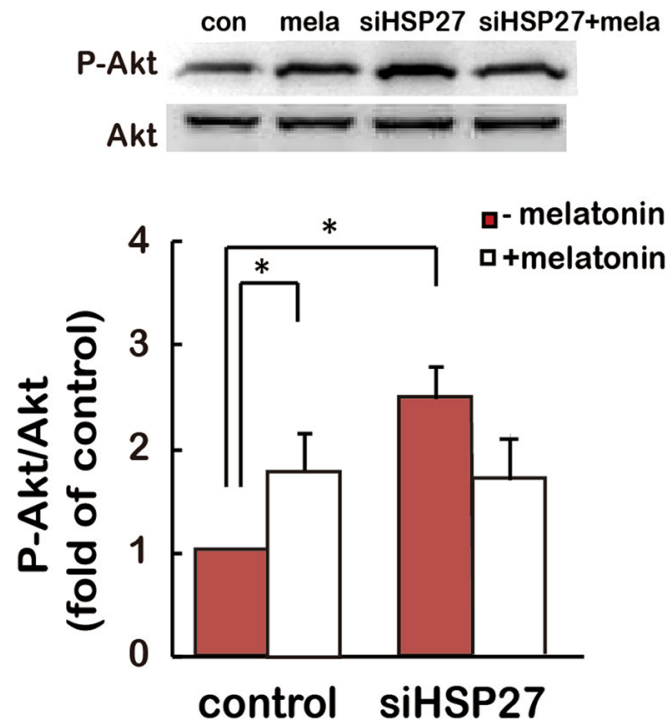
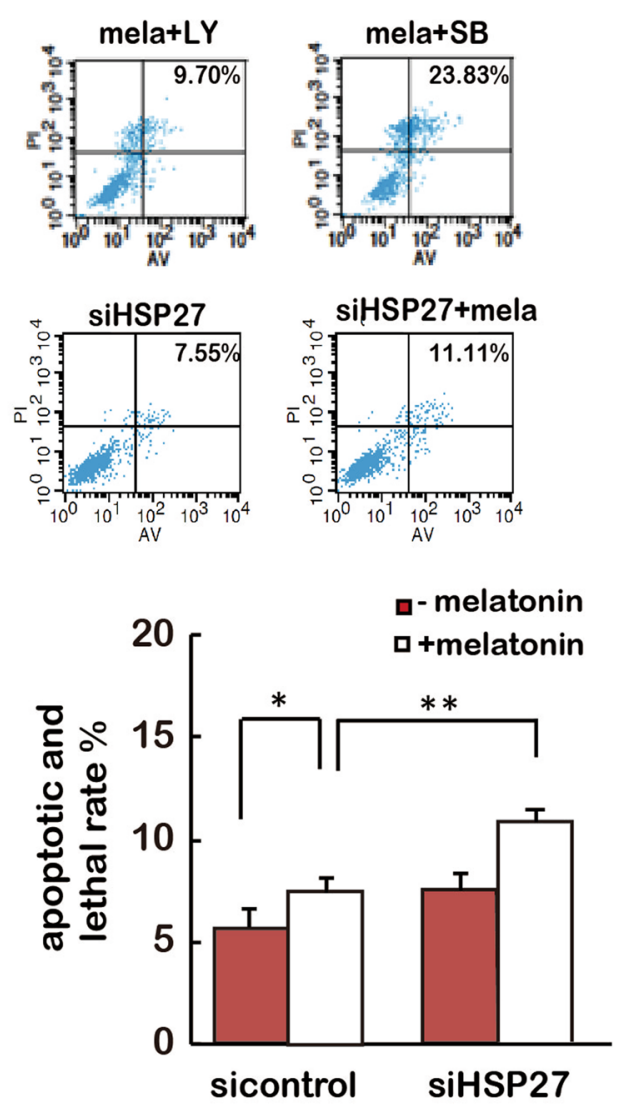

C
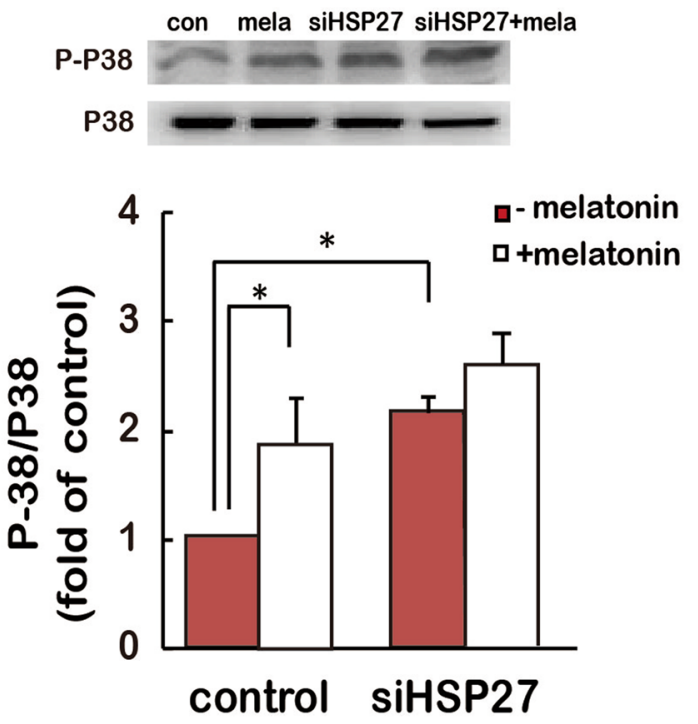

Fig. 5 Effects of SB203580, LY294002 and HSP27 silencing on melatonin-induced cell apoptosis. a Effects of SB203580, LY294002 and siHSP27 on melatonin stimulated cell apoptosis and necrosis were measured with FACS assay. ${ }^{*} \mathrm{P}<0.05$, ${ }^{*} \mathrm{P}<0.01$. b, c After incubation with melatonin for $24 \mathrm{~h}$, total protein extracts from SGC-7901 cells transfected with HSP27 siRNA or control siRNA were analyzed by immunoblotting for P-Akt (b) and P-P38 (c). *P $<0.05$ 
response to apoptotic stress in human prostate cancer cells [31]. However, in gastric cancer cells, whether P38 might affect the PI3K/Akt and HSP27 activation induced by melatonin is unknown. Here, our results clearly reveal that melatonin triggers a rapid stimulation of P38 activity. The selective P38 inhibitor SB203580 effectively blocks melatonin-stimulated PI3K/Akt and HSP27 activation. Consequently, we conclude that P38 is an upstream component of PI3K/Akt/HSP27 signaling pathway in melatonin-stimulated apoptosis in SGC-7901 gastric cancer cells.

Then another important question arises about the relationship between the activation of P38 and anti-apoptosis of PI3K/Akt/HSP27 after melatonin treatment. Accumulating evidence suggests that P38 could be strongly activated by environmental and genotoxic stresses [32]. Notably, P38 plays a dual role in stress-induced apoptosis. On the one hand, many chemotherapeutic agents including melatonin require P38 activity for the induction of apoptosis [33], on the other hand, the P38/HSP27 axis plays an important role in mediated drug resistance in cancer treatment [34]. Accordingly, we found that when P38 activity was blocked by its inhibitor, melatonin-stimulated cell apoptosis was dramatically increased, indicating that P38 activation is resistant to melatonin-induced apoptotic process. Adding to the results that P38 inhibitor could also suppress PI3K/Akt and HSP27 activation, these findings suggest that P38, through its regulation of PI3K/ Akt/HSP27 anti-apoptosis pathway, serves as a suppressor of melatonin-stimulated SGC-7901 cells apoptosis.

Intriguingly, in contrast to the well-established proapoptotic roles of melatonin, our study revealed clearly a distinguishable anti-apoptotic pathway induced by melatonin. Taken together, all these results demonstrate that HSP27 plays a crucial role in apoptotic resistance in melatonin-treated SGC-7901 gastric cancer cells, and its activation is most likely via the activation of P38/PI3K/ Akt signaling by melatonin. These findings are of potential pathophysiological importance for understanding the integration of melatonin-related signaling and further substantiate the molecular basis for clinical trials applying melatonin for the treatment of gastric cancer.

\section{Authors' contributions}

$J D$ and WD participated in the design of this study. WD, LG, YZ and YW carried out the study. WD, JC, BD performed the statistical analysis. JD wrote the manuscript. All authors read and approved the final manuscript.

\footnotetext{
Author details

${ }^{1}$ Cancer Center, Nanjing Medical University, 140 Hanzhong Road, Nanjing, Jiangsu 210029, China. ${ }^{2}$ Department of Physiology, Nanjing Medical University, 140 Hanzhong Road, Nanjing, Jiangsu 210029, China. ${ }^{3}$ Department of Biochemistry and Molecular Biology, Nanjing Medical University, 140 Hanzhong Road, Nanjing, Jiangsu 210029, China.
}

\section{Acknowledgements}

This work was supported by Grant from the National Natural Science Foundation of China (81372319), a Project Funded by Collaborative Innovation Center for Cancer Medicine; Jiangsu Key Lab of Cancer Biomarkers, Prevention and Treatment to Luo Gu, the National Natural Science Foundation of China (81201614), the Natural Science Foundation of Jiangsu province (BK2012839), the High-Level Talents in Six Industries of Jiangsu province (JY-020) and the Postdoctoral Science Foundation of China (2012T50511) to Jun Du, and a Project Funded by the Priority Academic Program Development of Jiangsu Higher Education Institutions (PAPD).

\section{Competing interests}

The authors declare that they have no competing interests.

Received: 8 August 2015 Accepted: 3 February 2016

Published online: 12 February 2016

\section{References}

1. Piazuelo MB, Correa P. Gastric cancer: overview. Colomb Med. 2013;44(3):192-201.

2. Torre LA, Bray F, Siegel RL, Ferlay J, Lortet-Tieulent J, Jemal A. Global cancer statistics, 2012. CA Cancer J Clin. 2015;65(2):87-108.

3. Xin Z, Jiang S, Jiang P, Yan X, Fan C, Di S, Wu G, Yang Y, Reiter RJ, Ji G. Melatonin as a treatment for gastrointestinal cancer: a review. J Pineal Res. 2015;58(4):375-87.

4. Jardim-Perassi BV, Arbab AS, Ferreira LC, Borin TF, Varma NR, Iskander AS, Shankar A, Ali MM, de Campos Zuccari DA. Effect of melatonin on tumor growth and angiogenesis in xenograft model of breast cancer. PLoS ONE. 2014;9(1):e85311.

5. Paroni R, Terraneo L, Bonomini F, Finati E, Virgili E, Bianciardi P, Favero G, Fraschini F, Reiter RJ, Rezzani R, et al. Antitumour activity of melatonin in a mouse model of human prostate cancer: relationship with hypoxia signalling. J Pineal Res. 2014;57(1):43-52.

6. Hong Y, Won J, Lee Y, Lee S, Park K, Chang KT. Melatonin treatment induces interplay of apoptosis, autophagy, and senescence in human colorectal cancer cells. J Pineal Res. 2014;56(3):264-74.

7. Bubenik GA. Gastrointestinal melatonin: localization, function, and clinical relevance. Dig Dis Sci. 2002;47(10):2336-48.

8. Tanaka T, Yasui Y, Tanaka M, Oyama T, Rahman KM. Melatonin suppresses AOM/DSS-induced large bowel oncogenesis in rats. Chem Biol Interact. 2009;177(2):128-36

9. Trivedi PP, Jena GB, Tikoo KB, Kumar V. Melatonin modulated autophagy and Nrf2 signaling pathways in mice with colitis-associated colon carcinogenesis. Mol Carcinog. 2016;55(3):255-67.

10. Katsogiannou M, Andrieu C, Rocchi P. Heat shock protein 27 phosphorylation state is associated with cancer progression. Front Genet. 2014;5:346.

11. Zhao M, Shen F, Yin YX, Yang YY, Xiang DJ, Chen Q. Increased expression of heat shock protein 27 correlates with peritoneal metastasis in epithelial ovarian cancer. Reprod Sci. 2012;19(7):748-53.

12. Yu Z, Zhi J, Peng $X$, Zhong $X, X u$ A. Clinical significance of HSP27 expression in colorectal cancer. Mol Med Rep. 2010;3(6):953-8.

13. Huang Q, Ye J, Chen W, Wang L, Lin W, Lin J, Lin X. Heat shock protein 27 is over-expressed in tumor tissues and increased in sera of patients with gastric adenocarcinoma. Clin Chem Lab Med. 2010;48(2):263-9.

14. Yang $Y X$, Sun $X F$, Cheng AL, Zhang GY, Yi H, Sun Y, Hu HD, Hu P, Ye F, Chen ZC, et al. Increased expression of HSP27 linked to vincristine resistance in human gastric cancer cell line. J Cancer Res Clin Oncol. 2009;135(2):181-9.

15. Hsu HS, Lin JH, Huang WC, Hsu TW, Su K, Chiou SH, Tsai YT, Hung SC. Chemoresistance of lung cancer stemlike cells depends on activation of Hsp27. Cancer. 2011;117(7):1516-28.

16. Du J, Zhang L, Yang Y, Li W, Chen L, Ge Y, Sun C, Zhu Y, Gu L. ATP depletion-induced actin rearrangement reduces cell adhesion via p38 MAPK-HSP27 signaling in renal proximal tubule cells. Cell Physiol Biochem. 2010;25(4-5):501-10.

17. Kanagasabai R, Karthikeyan K, Vedam K, Qien W, Zhu Q, llangovan G. Hsp27 protects adenocarcinoma cells from UV-induced apoptosis 
by Akt and p21-dependent pathways of survival. Mol Cancer Res. 2010;8(10):1399-412.

18. Turbov JM, Twaddle GM, Yang X, Liu NM, Murthy S. Effects of receptor tyrosine kinase inhibitor A47 on estrogen and growth factor-dependent breast cancer cell proliferation and apoptosis in vitro. J Surg Oncol. 2002;79(1):17-29.

19. Straume O, Shimamura T, Lampa MJ, Carretero J, Oyan AM, Jia D, Borgman CL, Soucheray M, Downing SR, Short SM, et al. Suppression of heat shock protein 27 induces long-term dormancy in human breast cancer. Proc Natl Acad Sci USA. 2012;109(22):8699-704.

20. Ebert MP, Schafer C, Chen J, Hoffmann J, Gu P, Kubisch C, Carl-McGrath S, Treiber G, Malfertheiner P, Rocken C. Protective role of heat shock protein 27 in gastric mucosal injury. J Pathol. 2005;207(2):177-84.

21. Evans J, Ko Y, Mata W, Saquib M, Eldridge J, Cohen-Gadol A, Leaver HA, Wang S, Rizzo MT. Arachidonic acid induces brain endothelial cell apoptosis via p38-MAPK and intracellular calcium signaling. Microvasc Res. 2015:98:145-58.

22. Leja-Szpak A, Jaworek J, Szklarczyk J, Konturek SJ, Pawlik WW. Melatonin stimulates HSP27 phosphorylation in human pancreatic carcinoma cells (PANC-1). J Physiol Pharmacol. 2007;58(Suppl 3):177-88.

23. Charette SJ, Lavoie JN, Lambert H, Landry J. Inhibition of Daxx-mediated apoptosis by heat shock protein 27. Mol Cell Biol. 2000;20(20):7602-12.

24. Chin YR, Toker A. Function of Akt/PKB signaling to cell motility, invasion and the tumor stroma in cancer. Cell Signal. 2009;21(4):470-6.

25. O'Shaughnessy RF, Welti JC, Cooke JC, Avilion AA, Monks B, Birnbaum MJ, Byrne C. AKT-dependent HspB1 (Hsp27) activity in epidermal differentiation. J Biol Chem. 2007;282(23):17297-305.

26. Li R, Li J, Sang D, Lan Q. Phosphorylation of AKT induced by phosphorylated Hsp27 confers the apoptosis-resistance in t-AUCB-treated glioblastoma cells in vitro. J Neurooncol. 2015;121(1):83-9.
27. Rane MJ, Pan Y, Singh S, Powell DW, Wu R, Cummins T, Chen Q, McLeish KR, Klein JB. Heat shock protein 27 controls apoptosis by regulating Akt activation. J Biol Chem. 2003;278(30):27828-35.

28. Zheng C, Lin Z, Zhao ZJ, Yang Y, Niu H, Shen X. MAPK-activated protein kinase-2 (MK2)-mediated formation and phosphorylation-regulated dissociation of the signal complex consisting of p38, MK2, Akt, and Hsp27. J Biol Chem. 2006;281 (48):37215-26.

29. Zhao B, Li X. Altholactone induces reactive oxygen species-mediated apoptosis in bladder cancer T24 cells through mitochondrial dysfunction, MAPK-p38 activation and Akt suppression. Oncol Rep. 2014;31(6):2769-75

30. Shin DY, Lee WS, Lu JN, Kang MH, Ryu CH, Kim GY, Kang HS, Shin SC, Choi YH. Induction of apoptosis in human colon cancer HCT-116 cells by anthocyanins through suppression of Akt and activation of p38-MAPK. Int J Oncol. 2009;35(6):1499-504

31. Kim J, Kang D, Sun BK, Kim JH, Song JJ. TRAIL/MEKK4/p38/HSP27/Akt survival network is biphasically modulated by the $\mathrm{Src} / \mathrm{CIN} 85 / \mathrm{c}-\mathrm{Cbl}$ complex. Cell Signal. 2013;25(1):372-9.

32. Han J, Lee JD, Bibbs L, Ulevitch RJ. A MAP kinase targeted by endotoxin and hyperosmolarity in mammalian cells. Science. 1994;265(5173):808-11.

33. Joo SS, Yoo YM. Melatonin induces apoptotic death in LNCaP cells via p38 and JNK pathways: therapeutic implications for prostate cancer. J Pineal Res. 2009;47(1):8-14.

34. Chen SF, Nieh S, Jao SW, Liu CL, Wu CH, Chang YC, Yang CY, Lin YS. Quercetin suppresses drug-resistant spheres via the p38 MAPK-Hsp27 apoptotic pathway in oral cancer cells. PLoS ONE. 2012;7(11):e49275.

\section{Submit your next manuscript to BioMed Central and we will help you at every step:}

- We accept pre-submission inquiries

- Our selector tool helps you to find the most relevant journal

- We provide round the clock customer support

- Convenient online submission

- Thorough peer review

- Inclusion in PubMed and all major indexing services

- Maximum visibility for your research

Submit your manuscript at www.biomedcentral.com/submit
() Biomed Central 